\title{
Expression of the visinin-like protein-1 in non-small cell lung carcinoma distinguishes
} squamous cell lung carcinoma from adenocarcinoma.

Shahan Mamoor, MS1

1'shahanmamoor@gmail.com

East Islip, NY USA

Non-small cell lung cancer, classified as adenocarcinomas or squamous cell lung carcinomas, is the major cause of cancer death in the United States and worldwide ${ }^{1}$. To understand the most significant transcriptional differences between adenocarcinomas and squamous cell lung carcinomas, we mined published microarray data from two separate studies ${ }^{2,3}$. We identified visinin-like protein-1 (VSNL1) as a distinguishing transcriptional feature of squamous cell lung carcinomas, suggesting the biology of VSNL1 may be relevant to the pathways critical for the development or maintenance of squamous cell lung carcinomas but not of adenocarcinomas. VSNL1 expression was significantly correlated with prognosis of patients with NSCLC, as patients with low tumor expression of VSNL1 possessed significantly longer median overall survival than those with high tumor expression of VSNL1. These analyses will also provide novel tools for diagnostic approaches and for guidance of treatment regimens for a cancer with dismal outlook.

Keywords: visinin-like protein-1, VSNL1, non-small cell lung cancer, adenocarcinoma, squamous cell lung carcinoma, systems biology of NSCLC, targeted therapeutics in NSCLC, targeted diagnostics in NSCLC. 
Non-small cell lung cancer is the single most common cancer in the United States and the most common cause of cancer death in the United States ${ }^{1}$. The two major types of nonsmall cell lung cancer are adenocarcinoma and squamous cell lung carcinoma ${ }^{4}$.

Treatment approaches and prognosis can differ based on whether a patient is diagnosed with adenocarcinoma or squamous cell lung carcinoma ${ }^{4}$, and current approaches to distinguishing adenocarcinoma from squamous cell lung carcinoma rely on time-consuming procedures involving immunohistochemical staining with multiple markers including TTF1, p40, p63, CK5 and/or CK7 and subsequent readout by pathologists who will proceed to label each section, in a binary fashion, as positive or negative for each marker ${ }^{5}$. To understand the molecular nature of ACC and SCLC tumors in an unbiased fashion and the systems level, and to facilitate discovery of genes with utility in NSCLC diagnostics in a quantitative real-time workflow providing a quantitative and independent measure rather than qualitative measure based on pathologist interpretation, we performed comparative transcriptome analysis ${ }^{2,3}$ of the two major types of NSCLC tumors: adenocarcinomas and squamous cell lung carcinomas.

This blind, systems-level approach identified the gene encoding visinin-like protein-1, VSNL1, as among the genes most differentially expressed when comparing adenocarcinomas and squamous cell lung carcinoma tumors, with VSNL1 expression distinguishing squamous cell lung carcinomas from adenocarcinomas, suggesting VSNL1 could be a useful tool in NSCLC diagnostics.

\section{Methods}

We utilized datasets GSE $74706^{2}$ and GSE335323 for this comparative transcriptome analysis of adenocarcinomas and squamous cell lung carcinomas. GSE74706 was generated using Agilent-026652 Whole Human Genome Microarray 4x44K v2 technology; for this analysis, we used $n=10$ adenocarcinomas and $n=4$ squamous cell lung carcinomas, and the analysis was performed using platform GPL13497. GSE33532 was generated using Affymetrix Human Genome U133 Plus 2.0 Array technology; for this analysis, we used $n=10$ adenocarcinoma tumors and $n=8$ squamous cell lung carcinomas, and the analysis was performed using platform GPL570.

The Benjamini and Hochberg method of $p$-value adjustment was used for ranking of differential expression but raw $p$-values were used to assess statistical significance of global differential expression. Log-transformation of data was auto-detected, and the NCBI generated category of platform annotation was used. A statistical test was performed to evaluate whether VSNL1 expression was significantly between adenocarcinoma and squamous cell carcinoma using a two-tailed, unpaired t-test with Welch's correction. We used PRISM for all statistical analyses of differential gene expression in NSCLC tumors (Version 8.4.0)(455). For Kaplan-Meier survival analysis, we used the Kaplan-Meier plotter online tool ${ }^{7}$ for correlation of VSNL1 mRNA expression levels with overall survival in $n=1925$ non-small cell lung cancer patients. 


\section{Results}

We mined published microarray data from two separate studies ${ }^{2,3}$ to describe in an unbiased fashion and at the systems-level genes whose expression was most specific to either one of the two major types of non-small cell lung cancer: adenocarcinomas and squamous cell lung carcinomas.

VSNL1 expression distinguishes squamous cell lung carcinomas from adenocarcinomas.

By comparing the global gene expression profiles of squamous cell lung carcinomas to adenocarcinomas, we found that the visinin-like protein-1 was among the most differentially expressed genes between the two major sub-types of NSCLC2 (Table 1). When sorting each of the genes expression by microarray based on significance of change in expression between adenocarcinomas and squamous cell lung carcinomas, VSNL1 ranked 4 out of 34183 total transcripts (Table 1). VSNL1 differential expression between adenocarcinomas and squamous cell lung carcinomas was statistically significant (Table $1 ; p=1.02 \mathrm{E}-11$ ).

In a separate dataset ${ }^{3}$, VSNL1 was again amongst the most differentially expressed when comparing squamous cell lung carcinomas to adenocarcinomas in NSCLC (Table 2). When sorting each of the genes expression by microarray based on significance of change in expression between adenocarcinomas and squamous cell lung carcinomas, VSNL1 ranked 25 out of 25906 total transcripts (Table 2). VSNL1 differential expression between adenocarcinomas and squamous cell lung carcinomas was again statistically significant (Table $2 ; p=1.07 \mathrm{E}-24)$.

Visinin-like protein-1 is expressed at significantly higher levels in squamous cell lung carcinomas than in adenocarcinomas.

We obtained exact mRNA values for VSNL1 to understand the magnitude and direction of difference in VSNL1 mRNA expression between adenocarcinomas and squamous cell lung carcinomas in NSCLC. VSNL1 was expressed at significantly higher levels in squamous cell lung carcinomas as compared to adenocarcinomas, and this difference was statistically significant (Figure 1 and Figure 2: $p<0.0001$ and $p<0.0001$, respectively). We calculated a mean fold change of $1.848 \pm 0.116$ in VSNL1 expression when comparing squamous cell lung carcinomas to adenocarcinomas (Table 2).

\section{Expression of VSNL1 is associated with patient survival in NSCLC.}

We performed Kaplan Meier survival analysis ${ }^{7}$ to determine if VSNL1 expression was correlated with patient outcomes in NSCLC. We found significant correlation between expression of VSNL1 and overall survival in patients with NSCLC (Figure 3 and Table 3); high VSNL1 tumor expression was a negative prognostic indicator. While median overall survival was 79.5 months for NSCLC patients with low expression of VSNL1, median overall survival was 62.2 months for NSCLC patients with high expression of VSNL1. Correlation of VSNL1 tumor expression with median OS with statistically significant (Figure 3; log rank $p$-value: 0.00093; hazard ratio: $1.24(1.09-1.4))$. 
Thus, by mining published microarray data from independent datasets, we found that VSNL1 was among the genes whose expression most significantly distinguished squamous cell lung carcinomas from adenocarcinomas in NSCLC. Moreover, we found a statistically significant correlation between VSNL1 tumor expression at the mRNA level and patient survival in NSCLC.

\section{Discussion}

To understand the most striking transcriptional features of each of the two major tumor types in NSCLC, we performed comparative transcriptome analysis of ACC and SCLC tumors using published microarray data, discovering visinin-like protein-1 as the among the most distinguishing transcriptional feature of squamous cell lung carcinomas.

The visinin-like protein-1 is the human homologue of the rat visinin-like peptide RNVP1 and is located on chromosome $2^{8}$. A (CA)n microsatellite repeat residing in the 3- untranslated region of VSNL1 is conserved between rat and human. VSNL1, also known as VILIP-1 is expressed in the brain, heart, testis and colon', as well as in the cardiac primordia during mouse development, in all four chambers of the adult mouse heart, and in the taste buds, cochlea, thryoid, tooth, salivary gland and adrenal gland during development ${ }^{10}$. VSNL1 is a member of the neuronal calcium sensor proteins that are post-translationally modified with a myristoyl group at the amino-terminus ${ }^{11}$. Following a depolarizing stimulus or glutamate receptor activation, VSNL1 could translocate in a reversible manner to membrane structures in hippocampal neurons, suggesting that VSNL1 could function as a stimulus-dependent calciummyristoyl switch ${ }^{11}$. In neurons of the hippocampus, VSNL1 can influence clathrin-mediated receptor recycling through control of cyclic GMP signaling ${ }^{12}$. VSNL1 is also expressed in the pancreatic islets and $\beta$-cells of the mouse pancreas, and ectopic expression of MIN6 $\beta$ cell line resulted in increased insulin secretion following glucose stimulation and as well as increased levels of cyclic AMP. Depletion of VSNL1 conversely decreased cAMP levels, as well as decreases in preproinsulin expression, basal insulin secretion and total cellular insulin levels ${ }^{13}$. VSNL1 is also expressed in the ureteric epithelium in the kidneys, and marks the ureteric bud tip in embryonic kidneys ${ }^{14}$. In mouse development, VSNL1 is required for early ureteric branching, and its expression is induced during ureteric budding and branching ${ }^{14}$. In mIMCD3 cells, VSNL1 suppresses canonical WNT pathway signaling as measured by activation of a TOPFLASH reporter in response to Wnt3A stimulation ${ }^{14}$.

In human cancers, VSNL1 was identified as a gene whose expression was significantly different between the tumors of colorectal cancer (CRC) patients with and without lymph node metastasis ${ }^{15}$. In stage III CRC, high expression of VSNL1 correlated with poorer prognosis and number of lymph node metastase ${ }^{15}$. In glioblastoma multiforme, VSNL1 was identified as a target of microRNA over-expressed in patient tumors and cell lines, miR-671-5p ${ }^{16}$. VSNL1 was found to be expressed at significantly higher levels in a highly-invasive sub-population of the SK-N-AS neuroblastoma cell line (HI) as compared to a low-invasive sub-population (LI), and both the rate of tumor growth and tumor volume of SK-N-AS HI cells was greater than that of SK-N-AS LI cells when xenografted in athymic nude mice ${ }^{17}$. When injected intravenously, lung metastases developed at markedly increased frequency in mice injected with SK-N-AS HI cells as compared to mice injected with SK-N-AS LI cells ${ }^{17}$. VSNL1 was expressed at higher levels in tumors from neuroblastoma patients with distant metastases as compared to patients without 
distant metastases ${ }^{17}$. Ectopic expression of VSNL1 was associated with increased anoikis potential of neuroblastoma cells and associated with expression of TrkB, ICAM1, MHC-I, CD44 and CD44v617. Thus, VSNL1 has been described as a stimulus-dependent calcium myristoyl switch in hippocampal neurons, marks the uretic bud tip in the embryonic kidney, can influence Wnt pathway signaling, and its expression is perturbed in colorectal cancer and neuroblastoma and appears to associate with metastasis and propensity to develop lymph node metastases and metastases to distant sites.

We found that the visinin-like protein-1 was the among the most differentially expressed gene when comparing the tumors of patients with the two most common sub-types of NSCLC: adenocarcinomas and squamous cell lung carcinomas. We also found significant correlation between overall survival of NSCLC patients and VSNL1 expression. VSNL1 has value as a diagnostic tool, as a prognostic indicator, and the biology of VSNL1 may be of value in understanding fundamental differences between the two major types of the most common type of cancer, and the most common cause of cancer death in the United States and worldwide. 


\section{References}

1. Siegel, R.L., Miller, K.D. and Jemal, A., 2019. Cancer statistics, 2019. CA: a cancer journal for clinicians, 69(1), pp.7-34.

2. Marwitz, S., Depner, S., Dvornikov, D., Merkle, R., Szczygieł, M., Müller-Decker, K., Lucarelli, P., Wäsch, M., Mairbäurl, H., Rabe, K.F. and Kugler, C., 2016. Downregulation of the TGF $\beta$ pseudoreceptor BAMBI in non-small cell lung cancer enhances TGF $\beta$ signaling and invasion. Cancer research, 76(13), pp.3785-3801.

3. Kabbout, M., Garcia, M.M., Fujimoto, J., Liu, D.D., Woods, D., Chow, C.W., Mendoza, G., Momin, A.A., James, B.P., Solis, L. and Behrens, C., 2013. Ets2 mediated tumor suppressive function and met oncogene inhibition in human non-small cell lung cancer. Clinical cancer research, 19(13), pp.3383-3395.

4. Cetin, K., Ettinger, D.S., Hei, Y.J. and D O'Malley, C., 2011. Survival by histologic subtype in stage IV nonsmall cell lung cancer based on data from the Surveillance, Epidemiology and End Results Program. Clinical epidemiology, 3, p.139.

5. Yuan, M., Huang, L.L., Chen, J.H., Wu, J. and Xu, Q., 2019. The emerging treatment landscape of targeted therapy in non-small-cell lung cancer. Signal Transduction and Targeted Therapy, 4(1), pp.1-14.

6. Osmani, L., Askin, F., Gabrielson, E. and Li, Q.K., 2018, October. Current WHO guidelines and the critical role of immunohistochemical markers in the subclassification of non-small cell lung carcinoma (NSCLC): Moving from targeted therapy to immunotherapy. In Seminars in cancer biology (Vol. 52, pp. 103-109). Academic Press.

7. Gyorffy, B., Surowiak, P., Budczies, J. and Lanczky, A., 2013. Online survival analysis software to assess the prognostic value of biomarkers using transcriptomic data in non-smallcell lung cancer. PloS one, 8(12), pp.e82241-e82241.

8. Polymeropoulos, M.H., Ide, S., Soares, M.B. and Lennon, G.G., 1995. Sequence characterization and genetic mapping of the human VSNL1 gene, a homologue of the rat visinin-like peptide RNVP1. Genomics, 29(1), pp.273-275.

9. Gierke, P., Zhao, C., Brackmann, M., Linke, B., Heinemann, U. and Braunewell, K.H., 2004. Expression analysis of members of the neuronal calcium sensor protein family: combining bioinformatics and Western blot analysis. Biochemical and biophysical research communications, 323(1), pp.38-43.

10.Ola, R., Lefebvre, S., Braunewell, K.H., Sainio, K. and Sariola, H., 2012. The expression of Visinin-like 1 during mouse embryonic development. Gene Expression Patterns, 12(1-2), pp. 53-62.

6 OF 13 
11.Spilker, C., Dresbach, T. and Braunewell, K.H., 2002. Reversible translocation and activitydependent localization of the calcium-myristoyl switch protein VILIP-1 to different membrane compartments in living hippocampal neurons. Journal of Neuroscience, 22(17), pp. 7331-7339.

12.Brackmann, M., Schuchmann, S., Anand, R. and Braunewell, K.H., 2005. Neuronal Ca2+ sensor protein VILIP-1 affects CGMP signalling of guanylyl cyclase $B$ by regulating clathrindependent receptor recycling in hippocampal neurons. Journal of cell science, 118(11), pp. 2495-2505.

13.Dai, F.F., Zhang, Y., Kang, Y., Wang, Q., Gaisano, H.Y., Braunewell, K.H., Chan, C.B. and Wheeler, M.B., 2006. The neuronal Ca2+ sensor protein visinin-like protein-1 is expressed in pancreatic islets and regulates insulin secretion. Journal of biological chemistry, 281(31), pp. 21942-21953.

14.Ola, R., Jakobson, M., Kvist, J., Perälä, N., Kuure, S., Braunewell, K.H., Bridgewater, D., Rosenblum, N.D., Chilov, D., Immonen, T. and Sainio, K., 2011. The GDNF target Vsnl1 marks the ureteric tip. Journal of the American Society of Nephrology, 22(2), pp.274-284.

15.Akagi, T., Hijiya, N., Inomata, M., Shiraishi, N., Moriyama, M. and Kitano, S., 2012. Visininlike protein-1 overexpression is an indicator of lymph node metastasis and poor prognosis in colorectal cancer patients. International journal of cancer, 131(6), pp.1307-1317.

16.Barbagallo, D., Condorelli, A., Ragusa, M., Salito, L., Sammito, M., Banelli, B., Caltabiano, R., Barbagallo, G., Zappalà, A., Battaglia, R. and Cirnigliaro, M., 2016. Dysregulated miR-671-5p/CDR1-AS/CDR1/VSNL1 axis is involved in glioblastoma multiforme. Oncotarget, 7(4), p.4746.

17.Xie, Y., Chan, H., Fan, J., Chen, Y., Young, J., Li, W., Miao, X., Yuan, Z., Wang, H., Tam, P.K. and Ren, Y., 2007. Involvement of visinin-like protein-1 (VSNL-1) in regulating proliferative and invasive properties of neuroblastoma. Carcinogenesis, 28(10), pp.2122-2130. 

when comparing whole transcriptomes of adenocarcinomas and squamous cell lung carcinomas, the two major types of non-small cell lung cancer.

Rank of differential expression, probe ID, $p$-value with respect to differential expression, $\mathrm{t}$, a moderated $\mathrm{t}$-statistic, $\mathrm{B}$, the log-odds of differential expression between the two groups compared, and gene are listed in this chart. 


\begin{tabular}{|c|c|c|c|c|c|c|c|}
\hline Rank & ID & p-value & $\mathbf{t}$ & B & FC & Gene & Gene name \\
\hline 25 & 203798_s_at & 1.07E-24 & 17.5955557 & 45.813366 & $1.848 \pm 0.116$ & VSNL1 & visinin like 1 \\
\hline
\end{tabular}

Table 2: Visinin-like protein-1 is among the most differentially expressed genes when comparing whole transcriptomes of adenocarcinomas and squamous cell lung carcinomas, the two major types of non-small cell lung cancer.

Rank of differential expression, probe ID, $p$-value with respect to differential expression, $\mathrm{t}$, a moderated t-statistic, $\mathrm{B}$, the log-odds of differential expression between the two groups compared, fold change of VSNL1 when comparing squamous cell lung carcinomas to adenocarcinomas, gene and gene name are listed in this chart. 


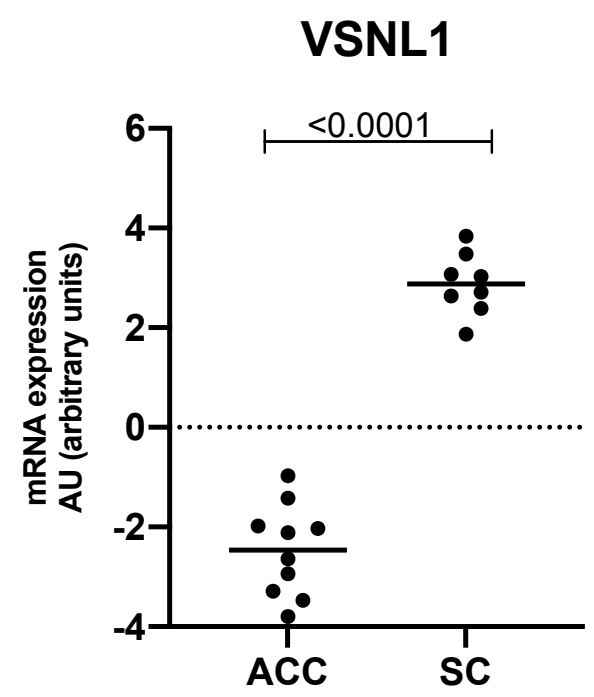

Figure 1: Expression of visinin-like protein-1 distinguishes squamous cell lung carcinoma from adenocarcinoma.

Messenger RNA (mRNA) levels of VSNL1 in the tumors of patients with adenocarcinoma ("ACC"; left) and in the tumors of patients with squamous cell lung carcinoma ("SC"; right) are graphically represented here with mean mRNA levels marked and the result of a statistical test evaluating significance of difference in mRNA expression between the tumors of patients with adenocarcinomas and squamous cell lung carcinomas, a $p$-value, listed above. 


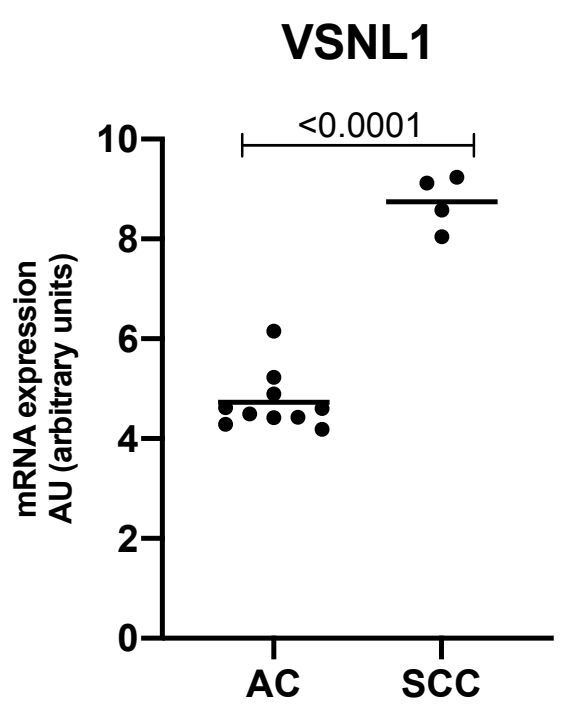

Figure 2: Expression of visinin-like protein-1 distinguishes squamous cell lung carcinoma from adenocarcinoma.

Messenger RNA (mRNA) levels of VSNL1 the tumors of patients with adenocarcinoma ("ACC"; left) and in the tumors of patients with squamous cell lung carcinoma ("SC"; right) are graphically represented here with mean mRNA levels marked and the result of a statistical test evaluating significance of difference in mRNA expression between the tumors of patients with adenocarcinomas and squamous cell lung carcinomas, a $p$ value, listed above. 


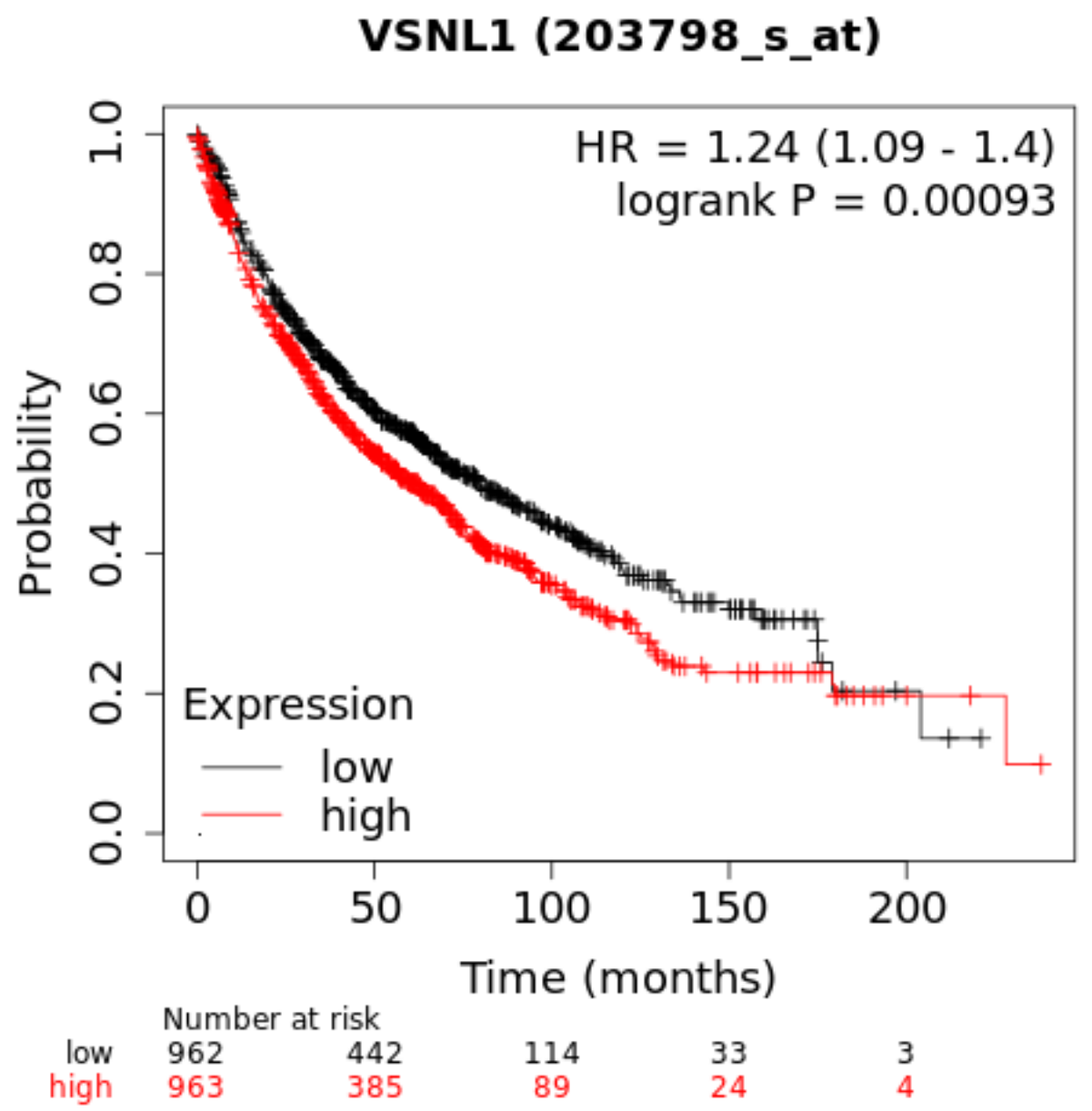

Figure 3: Visinin-like protein-1 expression in the tumors of patients with NSCLC correlates with overall survival.

Depicted in this Kaplan-Meier plot is the probability of overall survival for $n=1925$ total patients stratified into two groups, based on low or high expression of VSNL1 in patient tumors. The log rank $p$-value denoting statistical significance of difference in overall survival when comparing the two groups, as well as hazard ratio for this comparison is listed above. Listed below is the number of patients at risk (number of patients alive) per interval, after stratification based on VSNL1 expression; in the first interval, number at risk is number of patients alive; in each subsequent interval, number at risk is the number at risk less those who have expired or are censored. 
